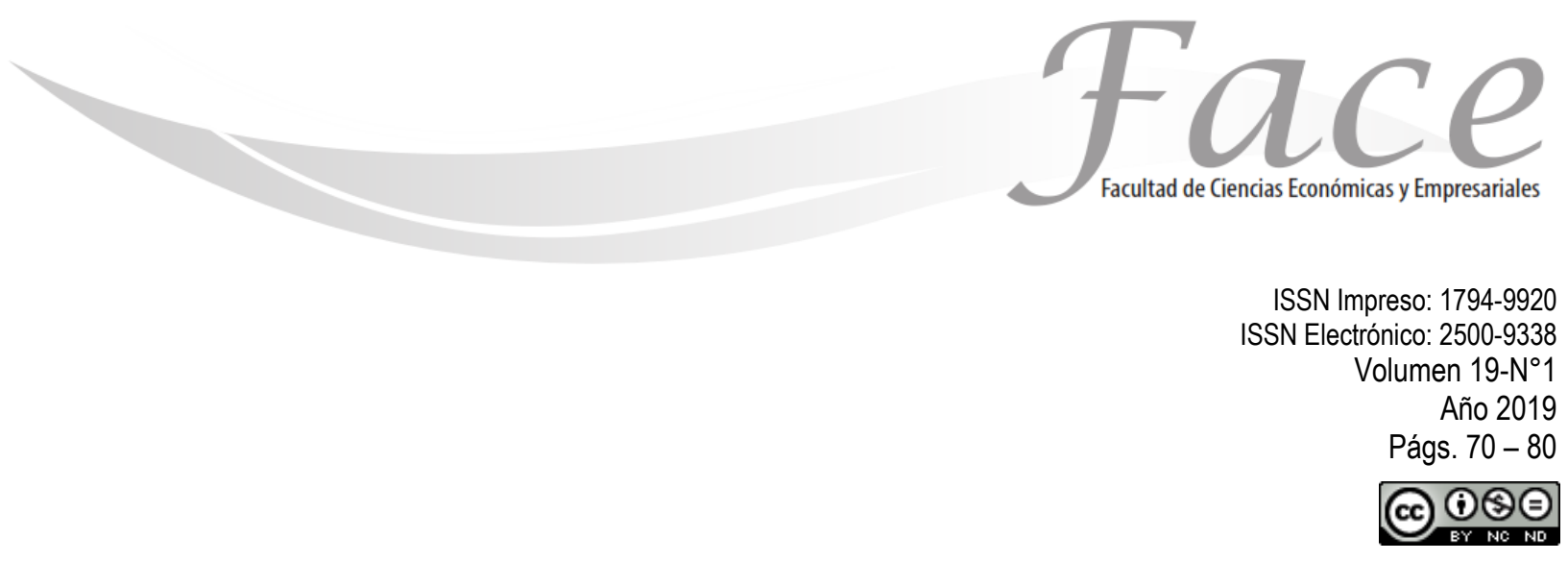

\title{
SIMULACIÓN DINÁMICA DEL PRONÓSTICO Y MERCADOTECNIA DE UN PRODUCTO PARA CONTROLAR EL RIESGO INHERENTE INTEGRANDO EL VALOR PRESENTE NETO
}

\author{
Jaime Eduardo Gutiérrez Ascón * \\ ORCID: https://orcid.org/0000-0003-4065-3359
}

Fecha de Recepción: Enero 26 de 2019

Fecha de Aprobación: Abril 14 de 2019

\section{Resumen:}

Esta investigación busca asegurar el lanzamiento de la Televisión Interactiva (ITV) como nuevo producto en el mercado televisivo; donde, asegurando con el mejor pronóstico el mejor Valor Actual Neto que garantiza la inversión inicial de implementación. Los especialistas consideran una proyección de crecimiento del mercado para los años 2015 en adelante de 50000 hogares con televisión por año y se espera llegar a 3450000 en el año 2020 con un Valor Actual Neto de \$63 883374 para la inversión inicial \$110 000 000. Este pronóstico es ajustado por el simulador Cristal Ball (CB) de 3295768 hogares que consumen televisión para el año pronosticado 2015 hasta los 3581193 para el año 2020 con el Valor Actual Neto (VPN o VAN) neto de \$ 73715279 con la misma tasa de descuento del 10\%. El proceso simula 12500 pruebas alcanzando el nivel del $95 \%$ de confianza nivel alcanzado en solo 12 437 pruebas. Con el nuevo producto (ITV) insertado en el mercado según el análisis de sensibilidad, se encuentra que el ITV contribuirá con el 28,8\% del Valor Presente Neto en el año 2020 y como es de esperar, la contribución negativa de la inversión inicial aporta negativamente con 13,7\% al VPN. El nuevo Valor Actual Neto crece en aproximadamente diez millones como resultado del proceso cuantitativo simulado.

Palabras clave: Pronóstico, Demanda, Simulación, Valor Actual Neto

\footnotetext{
*Ingeniero Industrial-. Universidad Nacional José Faustino Sánchez Carrión, Huacho- PERÚ- Ingeniero Industrial-. Perú. Contacto: ing_jagu@hotmail.com
} 


\title{
DYNAMIC SIMULATION OF THE PROGNOSIS AND MARKETING OF A PRODUCT TO CONTROL THE INHERENT RISK INTEGRATING THE NET PRESENT VALUE
}

\begin{abstract}
:
This research seeks to ensure the launch of Interactive Television (ITV) as a new product in the television market; where, ensuring with the best forecast the best Net Present Value that guarantees the initial investment of implementation. The specialists consider a projection of growth of the market for the years 2015 onwards of 50000 households with television per year and it is expected to reach 3450000 in the year 2020 with a Net Present Value of \$ 63883374 for the initial investment \$ 110 000 000. This forecast is adjusted by the Cristal Ball (CB) simulator of 3295768 households that consume television for the forecast year 2015 to 3581193 for the year 2020 with Net Present Value (NPV or NPV) net of \$ 73715279 with the same 10\% discount rate. The process simulates 12500 tests reaching the level of $95 \%$ confidence level reached in only 12,437 tests. With the new product (ITV) inserted in the market according to the sensitivity analysis, it is found that the ITV will contribute with $28.8 \%$ of the Net Present Value in the year 2020 and as expected, the negative contribution of the initial investment contributes negatively with $13.7 \%$ to the VPN. The new Net Present Value grows by approximately ten million as a result of the simulated quantitative process.
\end{abstract}

Keywords: Forecast, Demand, Simulation, Net Present Value

\section{SIMULAÇÃO DINÂMICA DO PROGNÓSTICO E MARKETING DE UM PRODUTO PARA CONTROLAR RISCO INERENTE POR INTEGRAR O PRESENTE VALOR LÍQUIDO}

Resumo:

Esta pesquisa visa garantir o lançamento da Televisão Interativa (ITV) como um novo produto no mercado de televisão; onde, garantindo com a melhor previsão, o melhor Valor Presente Líquido que garanta 0 investimento inicial de implementação. Especialistas acreditam que um crescimento do mercado projetada para os anos de 201550000 lares com TV por ano e deverá atingir 3,45 milhões em 2020, com um valor presente líquido de US $\$ 63.883 .374$ para o investimento de US $\$$ inicial 110000 000. Esta previsão é ajustado pelo simulador Crystal Ball (CB) de 3,295,768 famílias que consomem televisão para 0 ano de previsão de 2015 até 3581193 2020, com valor presente líquido líquido (VPL ou VPL) \$ 73715279 com a mesma taxa de desconto de $10 \%$. O processo simula 12500 testes atingindo o nível de $95 \%$ de confiança atingido em apenas 12.437 testes. Com o novo produto (ITV) inserido no mercado como análise de sensibilidade, é que ITV contribuirá $28,8 \%$ do valor presente líquido em 2020 e, como esperado, o contributo negativo do investimento inicial Contribui negativamente com 13,7\% para a VPN. O novo Valor Presente Líquido cresce em aproximadamente dez milhões como resultado do processo quantitativo simulado.

Palavras-chave: Previsão, Demanda, Simulação, Valor Presente Líquido. 


\section{INTRODUCCIÓN:}

Las empresas contemporáneas están siempre buscando mejores alternativas para satisfacer las necesidades del mercado demandante; es por tanto necesario planear en términos de las nuevas necesidades de entrega de productos 0 servicios que cumplan las expectativas. El mercado tecnológico y su desarrollo en tecnologías de información están abriendo un nuevo mercado con la llamada televisión interactiva (ITV) donde los usuarios puedan programar sus películas, deportes, entrenamiento o mejor aún educación. Sin embargo, las empresas prestadoras de servicio de televisión como en general las de telecomunicaciones deben enfrentar interrogantes que no son tan simples de absolver, más grave aun cuando estas decisiones colocan en juego el capital de la empresa o la imagen o prestigio cuando un nuevo producto es insertado en el mercado y éste no recibió el tratamiento adecuado para controlar la incertidumbre y su consecuencia directa el riesgo. Cuando los modelos matemáticos acuden en auxilio de la toma de decisiones de los empresarios, corren la buena o mala suerte de salir bien librados de las aventuras empresariales.

Los tiempos actuales exigen que los procesos 0 modelos matemáticos analíticos con que se cuentan en las empresas sean utilizados con muchísima reserva en la toma de decisiones y quienes deciden invertir en un nuevo producto deben saber que los avances tecnológicos y programas con que contamos en estos tiempos deben aproximarnos a los valores o parámetros que nos den cierta confianza en lo que nos depara el futuro. Se afirma entonces que el futuro no se puede conocer; sin embargo, los profesionistas de estos tiempos no tenemos excusa alguna para presentar disculpas por el fracaso de una inversión. Además, se afirma que el futuro es ahora y debemos estar preparados, debemos conocer los procesos dinámicos que nos permitan repetir el lanzamiento de un nuevo producto al mercado y no esperar que transcurra "n" periodos de tiempo para darnos cuenta que "nos pudo ir mejor" si hubiéramos tomado mejores decisiones.

Es preciso partir de los pronósticos. Hasta ahora, los paquetes o programas estadísticos con que disponemos para procesar la información, sin temor a equivocarnos, no son suficientes toda vez que debemos recolectar data histórica y aproximar en una suerte de prueba el valor futuro pronosticado estadísticamente. En la toma de decisiones, cuando no es posible contar con información para aproximarnos lo mejor posible al siguiente valor " $n+1$ " por llegar en el siguiente año, se dispone de las consultas a los expertos 0 especialistas en el tema quienes nos indicarán ( siempre en base a su experiencia) lo que está por suceder; este método llamado Método Delphi tiene sus limitaciones, este oráculo humano tiende a veces a equivocarse porque sencillamente son seres humanos y basan sus análisis en la experiencia desde su óptica personal y vivencial; por otro lado, la experiencia de un mercadólogo de México no será la misma que un mercadólogo europeo ni mucho menos comparado con un CEO de los países llamados Tigres del Asia.

A partir de este punto, nos gustaría saber antes de realizar las inversiones para el lanzamiento de un nuevo producto al mercado de la televisión si el nuevo producto llamado televisión interactiva (ITV) tiene potencial de éxito en la comunidad consumidora sabiendo que existen ya en el mercado la TV por cable, la TV por antena parabólica y la señal de TV tradicional. La cultura de consumir televisión está migrando hacia nuevos productos que permitan al consumidor aquella televisión que sea capaz de entretener con deportes, noticias, educación; pero cuando el hombre llega del trabajo a su casa, los niños llegan del colegio y en general las familias se reúnen para disfrutar este entretenimiento llamado la televisión resulta que a la hora que el cliente lo necesita no lo encuentra, sucede entonces que las emisoras programan y según criterio, tratando de insertarse 
en las "horas pico de teleaudiencia" por el tema de los auspiciadores televisivos.

Por otro lado, debemos conocer y controlar la tasa de crecimiento demográfico de las comunidades, de los consumidores específicamente distribuidos en las cuotas de mercado. Ya el panorama se va complicando y las variables van tomando posiciones críticas para la toma de decisiones, por ejemplo que pasará con la cuota de clientes del nuevo producto en los siguientes uno, dos, tres, cuatro, cinco 0 seis años conociendo bien que a medida que nos alejamos de la fecha límite de la data histórica es más incierto el panorama. Hasta aquí, es la incertidumbre de lo que no sabemos qué pasará mañana lo que nos paraliza al momento de tomar decisiones de inversión de capital.

Si los expertos del oráculo humano llamados especialistas 0 expertos nos dicen que esperamos una tasa de crecimiento de consumidores de un producto que no es conocido aun en el mercado y afirman por ejemplo que se espera una tasa de crecimiento aproximadamente de 50000 usuarios del nuevo producto de televisión interactiva (ITV) honestamente no estará en condiciones de responder por el fracaso si la proyección establecida por los expertos es correcta. Lo que si tiene sentido es afirmar críticamente que los gastos se pueden incrementar progresivamente cuando el proyecto de instalación de la red de ITV se extiende en el territorio.

Hasta este punto, son demasiadas conjeturas que los profesionistas deben considerar al momento de tomar decisiones; aún falta la interrogante más compleja y complicada de responder en este escenario de incertidumbres descritas; es, cuanto es la tasa de descuento TIR en que recuperaremos la inversión de insertar un nuevo producto de televisión en el mercado y la siguiente pregunta, cual es el Valor Presente Neto VPN ( o también Valor Actual Neto VAN) de todo el balance financiero de ingresos y egresos de este proyecto de inversión de un nuevo producto.
Si los tomadores de decisiones, sean éstos de cualquier especialidad del conocimiento como economistas, administradores, ingenieros, mercadólogos ya tienen serias dificultades para tomar decisiones, hay que considerar el enfoque desde el punto de vista de los accionistas de la empresa de televisión. Son ellos quienes tienen interrogantes muchísimo más serias, complejas y complicadas de responder en una toma de decisiones empresariales. Una pregunta capital y hasta ahora casi no existe seguridad de la respuesta es por ejemplo la siguiente: ¿Cuál es el nivel de confianza para estimar las utilidades del proyecto en digamos 20 millones y 80 millones de dólares? ¿Cuál es la probabilidad de percibir utilidades a lo más de 56 millones? 0 tal vez pregunten sobre la probabilidad de percibir utilidades mayores a 40 millones? Pero la realidad nos indica que los accionistas o dueños del negocio no se detendrán con las preguntas al momento de tomar estratégicamente lanzar un nuevo producto al mercado porque así lo han percibido los expertos de la empresa de televisión. ¿Cuáles son los factores clave de éxito que impulsan el rendimiento financiero del nuevo producto? Y todavía más: ¿En cuánto aportan estos factores clave de éxito a las utilidades del proyecto?

Hasta aquí, son solo preguntas, y se exige que se dispongan de modelos para dar respuesta a estas interrogantes. Los tecnólogos de estos tiempos disponemos de procesos dinámicos de simulación estocástica para repetir miles de veces (pruebas) y determinar el mejor pronóstico, el mejor Valor Actual Neto VAN del proyecto pronosticado con el Oráculo Delphi a una tasa interna de retorno del $10 \%$ se obtiene un VAN de aproximadamente $\$ 63$ 883374 y los aportes porcentuales de aquellos factores de éxito del proyecto; el oráculo a emplear es Cristal Ball (CB). 
Por otro lado, para realizar un pronóstico, es preciso considerar que la información considerada como data histórica esta elaborada según sean los periodos horas, dias, semanas, meses, años seas que se traten de pronósticos cualitativos o cuantitativos. La serie de tiempo tiene dos componentes que son a saber la tendencia y la estacionalidad, donde el componente de tendencia es básicamente la pendiente que toma la data histórica, la misma que puede ser positiva ( crecimiento) negativa ( decrecimiento) 0 pendiente cero ( $\sin$ cambio en el tiempo).

"Cuando las series de tiempo tienen un componente ciclico que caen de manera regulkar abajo y arriba de la linea de tendencia se considera que la data histórica tiene estacionalidad" (Anderson, 2008, p. 769)

2.2 Simulación. Según afirma (Winston, 2005) en su libro Investigación de Operaciones, afirma que " La simulación es una técnica de las ciencias administrativas muy poderosa y se utiliza mucho para el análisis y estudio de sistemas complejos" Debemos considerar que el modelo de simulación una abstracción de la realidad en donde la lógica de las suposiciones entre los objetos de interés del sistema constituye el modelo. Los eventos a simular en el mundo real pueden ser discretos (frecuencia o conteo) 0 por otro lado continuos (medición) y la arquitectura del modelo de simulación toma como elementos de soporte a los números aleatorios de Monte Carlo para garantizar de esta manera que el sesgo ( valores determinados ) no afecte los resultados; esta aleatoriedad que el programa considera, asegura que sean posibles resultados probabilísticos o también llamados estocásticos para la simulación en el modelo del mundo real.

"Debemos considerar que el procedimiento de generar por ejemplo tiempos en una línea de espera o como en nuestro caso, la frecuencia o cantidad de hogares con TV generados a partir de las distribuciones de probabilidad, se conoce como muestreo de distribuciones de probabilidad o generación de variables aleatorias o bien, muestreo de Monte Carlo" (Winston, 2005, p. 1153)

2.3. Valor Presente Neto. Según (Monks, 1991) en su obra Administración de Operaciones afirma que:

El Valor Presente Neto o también llamado Valor Actual Neto indica el valor de un ingreso futuro 0 un gasto en 
términos de unidades monetarias traídos al presente, en donde estos flujos de efectivo generalmente representan el valor de la inversión, los costos de operación y mantenimiento y los ingresos

2.4 Método de Monte Carlo. Fue propuesto por J. Von Neumann, al respecto, se afirma que es una técnica de selección de números aleatorios a través de una o más distribuciones de probabilidad para utilizar estos números en una simulación creando un universo teórico que es descrito por la ley de probabilidad que genera un universo de números del cual se obtiene una muestra, se emplean para aproximar expresiones matemáticas analíticas complejas y costosas alcanzando exactitud. Los resultados se esperan que sean coincidentes cuando se utilizan los valores semilla generalmente de uno al seis. Su nombre se debe al casino Monte Carlo localizado en el Principado de Mónaco, ciudad considerada la capital del juego al azar.

Estos números son empleados para efectuar simulaciones en diferentes áreas o sectores del quehacer humano, desde la evaluación de proyectos, energía, hasta las finanzas. Este método permite al usuario conocer la probabilidad con la que un evento puede ocurrir, sustituyendo valores iniciales por un valor determinado y se calculan finalmente los valores finales produciendo así distribuciones de valores de posibles resultados.

2.5 Distribuciones de probabilidad. Una distribución es un conjunto de resultados posibles de un experimento estadístico junto a su probabilidad asociada, donde la función de probabilidad $\mathrm{P}(\mathrm{x})$ mide la probabilidad de cada uno de los valores que toma la variable aleatoria. Se deben cumplir las siguientes leyes: $0 \geq P(x) \leq 1$ y $\quad \sum P(x)=1$

Distribución Normal. Es una de las más empleadas, donde su principal característica es que es simétrica y asintótica, se calcula sobre variables aleatorias continuas las que son obtenidas por proceso de medición siendo sus valores exactos o decimales. Sus parámetros son la media y la desviación estándar. Esta distribución es también llamada Curva de la Campana o Curva Gaussiana,

Distribución Triangular: Es utilizada cuando se conocen los valores mínimo y máximo y además el más probable. Distribución Uniforme: Todos los valores entre el mínimo y el máximo tienen la misma probabilidad de producirse como por ejemplo costos y alquileres. Distribución Logarítmico Normal: Utilizada en gran número de situaciones en que los valores se sesgan positivamente en donde la mayoría de los valores están cerca del valor mínimo, generalmente utilizada en análisis financieros. Distribución Beta: Es muy flexible para modelar probabilidades en estadística bayesiana, sus parámetros son mínimo, máximo, alfa y beta. Distribución Beta PERT: Derivada de la distribución beta, es utilizada en análisis de riesgo de proyectos para duración o costos de tareas. Distribución Gamma: Se puede considerar como una distribución de la cantidad de tiempo hasta la aparición de un evento "r" en un evento Poisson.

El programa Cristal Ball considera veintidós distribuciones para realizar las simulaciones.

\section{METODOLOGÍA:}

Para desarrollar el presente trabajo, se deben considerar algunas de las premisas fundamentales como la hoja de cálculo electrónica donde se considera el flujo de caja del proyecto de inversión para el nuevo producto ITV en el mercado. Considerar la tabla 01 tomando en cuenta que están identificado seis períodos del pronóstico desde el año 2015 al 2020, los cuatro productos de televisión y las cuotas de mercado en 50000 hogar en que usarían ITV en la zona geográfica:

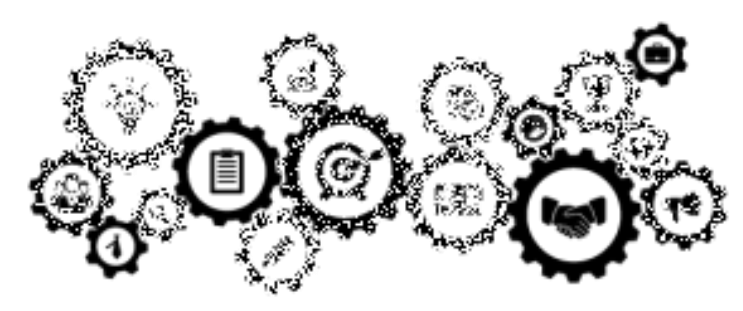


Tabla 01: Cuota de Mercado

\begin{tabular}{lccrrrr} 
& \multicolumn{1}{c}{ año } & \multicolumn{1}{c}{ año } & \multicolumn{1}{c}{ año } & \multicolumn{1}{c}{ año } & \multicolumn{1}{c}{ año } & \multicolumn{1}{c}{ año } \\
& \multicolumn{1}{c}{2015} & \multicolumn{1}{c}{2016} & \multicolumn{1}{c}{2017} & \multicolumn{1}{c}{2018} & \multicolumn{1}{c}{2019} & \multicolumn{1}{c}{2020} \\
\cline { 2 - 8 } & 3200000 & 3250000 & 3300000 & 3350000 & 3400000 & 3450000 \\
Hogares con TV & & & & & & \\
Cuota de mercado & & & & & & \\
TV Cable & 0.78 & 0.74 & 0.7 & 0.6 & 0.54 & 0.47 \\
Antena parabólic & 0.12 & 0.14 & 0.16 & 0.18 & 0.2 & 0.22 \\
Señal de TV & 0.08 & 0.07 & 0.06 & 0.05 & 0.04 & 0.03 \\
TV Interactiva (I & 0.02 & 0.05 & 0.08 & 0.17 & 0.22 & 0.28 \\
\hline TOTAL & $100 \%$ & $100 \%$ & $100 \%$ & $100 \%$ & $100 \%$ & $100 \%$
\end{tabular}

Por otro lado, en la Tabla 02 se presenta el tamaño de mercado de hogares que usarían ITV:

Tabla 03: Ingresos por ITV

Modelo de Pronóstico de Producto: ITV y VPN de inversiones.

\begin{tabular}{|c|c|c|c|c|c|c|}
\hline & $\begin{array}{c}\text { año } \\
2015\end{array}$ & $\begin{array}{c}\text { año } \\
2016\end{array}$ & $\begin{array}{c}\text { año } \\
2017\end{array}$ & $\begin{array}{c}\text { año } \\
2018\end{array}$ & $\begin{array}{c}\text { año } \\
2019\end{array}$ & $\begin{array}{c}\text { año } \\
2020\end{array}$ \\
\hline \multicolumn{7}{|c|}{ Tamaño de Mercado } \\
\hline TV Cable & 2496000 & 2405000 & 2310000 & 2010000 & 1836000 & 1621500 \\
\hline Antena paral & 384000 & 455000 & 528000 & 603000 & 680000 & 759000 \\
\hline Señal de TV & 256000 & 227500 & 198000 & 167500 & 136000 & 103500 \\
\hline TV Interacti & 64000 & 162500 & 264000 & 569500 & 748000 & 966000 \\
\hline TOTAL & 3200000 & 3250000 & 3300000 & 3350000 & 3400000 & 3450000 \\
\hline
\end{tabular}




\section{SIMULACIÓN DINÁMICA DEL PRONÓSTICO Y MERCADOTECNIA DE UN PRODUCTO PARA CONTROLAR EL RIESGO INHERENTE INTEGRANDO EL VALOR PRESENTE NETO \\ Jaime Eduardo Gutiérrez Ascón}

Consecutivamente, empleando la plataforma del software Cristal Ball se presenta la tabla completa donde se definen la Decisión, la Suposición (celdas color verde) y la Previsión (celda color celeste) de las variables que previamente se han considerado como las de trabajo para la simulación.

La celda resultante de pronóstico es la que resulta de ecuaciones o fórmulas de cálculo de las celdas de suposición de este modelo.

Igualmente, se consideran la "semilla raíz" generadora de los números aleatorios, generalmente se definen en la secuencia $1,2,3,4,5$, y 6 esta serie garantiza que realizando distintas corridas del programa resulten los mismos resultados aun cuando la ley de aleatoriedad de los números Monte Carlo por definición, nunca un número es similar a otro.
Igualmente se define la velocidad a la que debe realizarse la simulación para esto, se define el número de veces que se realiza la simulación. Para nuestro caso consideraremos realizar la simulación doce mil quinientas veces (12 500) y estableceremos los pronósticos tomando en cuenta la data histórica desde el año 1995 hasta el año 2014 y de aquí en adelante el periodo a simular desde el año 2015 hasta el 2020. La hoja de calculo de CB se presenta en la siguiente tabla con el VAN de inicio de simulación. Seis periodos y pronósticos estimados de 50000 incrementados anualmente.

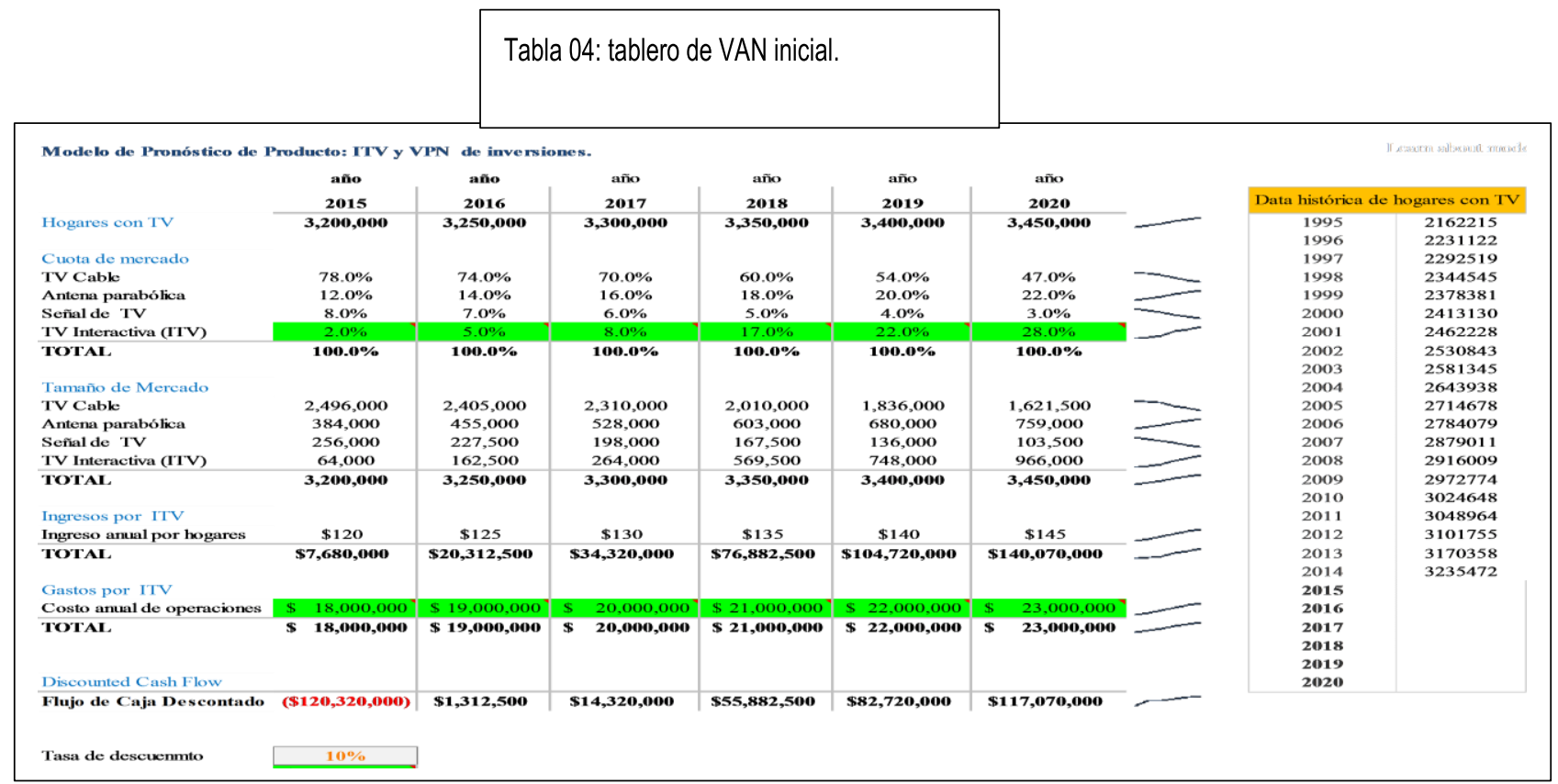


ISSN: 1794-9920 Impreso / Electrónico 2500-9338

Enero - Julio de 2019 - Volumen 19 Número 2, Año 2019 Págs. 70 - 80

\section{Hallazgos}

Figura 01: Reporte de pronóstico. Cristal Ball.

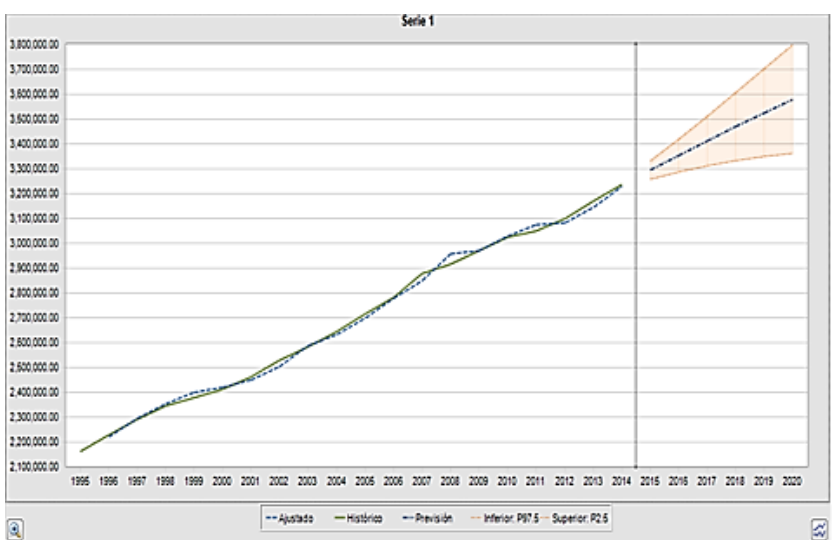

Según este reporte de salida de $\mathrm{CB}$, no existe estacionalidad de la data histórica de los hogares con TV desde el año 1995 hasta el año 2014.El método ARIMA proceso autorregresivo de la media móvil es mejor valor determinado automáticamente $(1,1,1)$ este parámetro asegura el mejor pronóstico hasta este momento.Las estadísticas de la data histórica se presentan en el lado derecho del reporte. Se ha definido el período de previsión de seis (años 2015 al 2020).
Ahora los resultados después de realizado el pronóstico para el periodo 2015 a 2020 y considerado el nuevo VAN

Por otro lado se presentan los resultados de la simulación para determinar los intervalos de probabilidad a los cuales se esperan los nuevos valores actuales netos VAN

Existe una probabilidad de aproximadamente el $70 \%$ que el valor Actual Neto se encuentre entre 20 millones y 80 millones

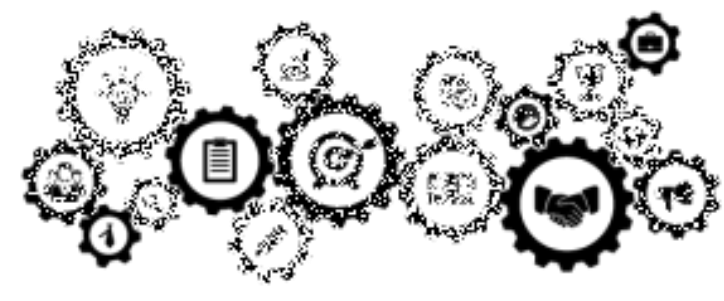

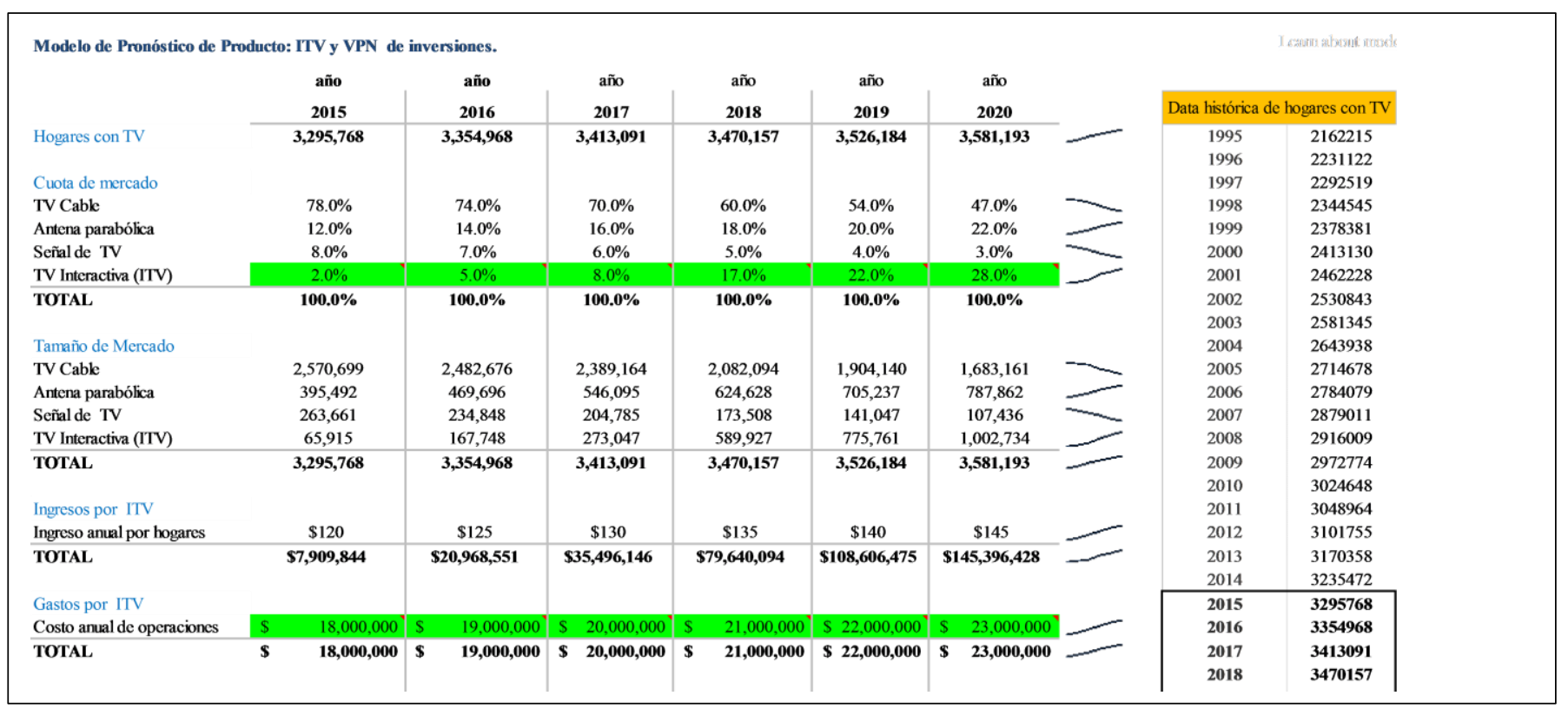


Figura 02: Reporte simulación nuevo VAN

Ahora los resultados después de realizado el pronóstico para el periodo 2015 a 2020 y considerado el nuevo VAN

Por otro lado se presentan los resultados de la simulación para determinar los intervalos de probabilidad a los cuales se esperan los nuevos valores actuales netos VAN

Existe una probabilidad de aproximadamente el $70 \%$ que el valor Actual Neto se encuentre entre 20 millones y 80 millones

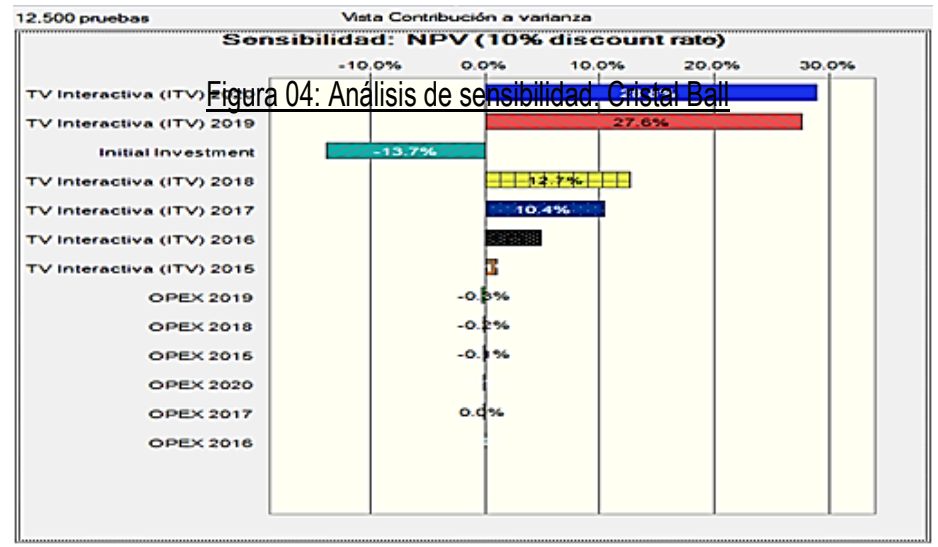

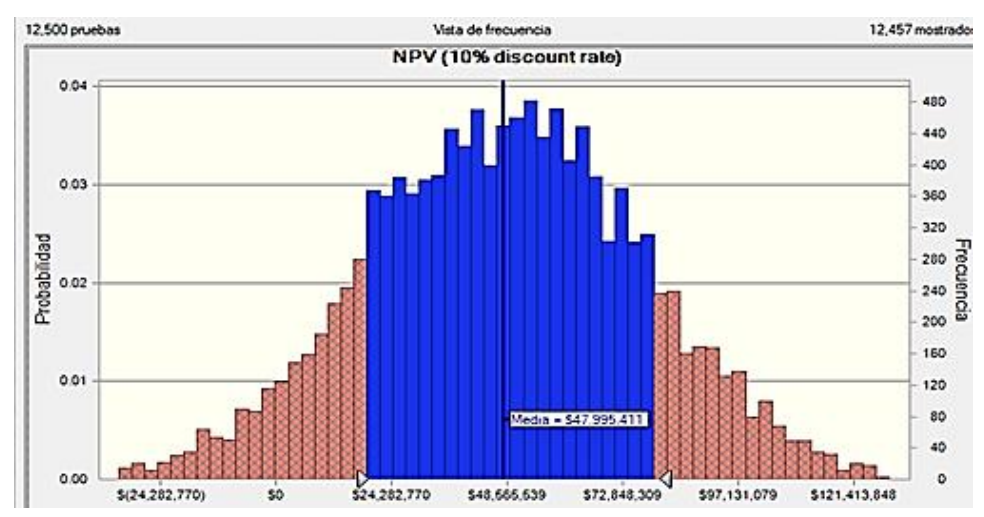

Figura 03: Reporte VAN simulado. Cristal Ball.

La sensibilidad está determinada por las variables que impactan directa 0 indirectamente el VAN final pronosticado 12500 veces y se encuentra que en el periodo de los años 2019 y 2020 se registra un aporte de $56.4 \%$ en el VAN

\section{CONCLUSIONES:}

Según el pronóstico de los especialistas, para los años 2015 al 2020 la data histórica de hogares con TV es conservadora con 19950000 comparada con la pronosticada por CB son 20641361 hogares con TV el diferencial de 691361 hogares afectan las utilidades en esta decisión de marketing.

2. Los especialistas consideran que las utilidades netas del negocio cuando se lanza un nuevo servicio de televisión al mercado son del orden de $\$ 150985000$ valor comparado con el pronóstico realizado por $\mathrm{CB}$, las utilidades son \$ 165017538 con un diferencial de \$ 14 032538 .

3. El Valor Actual Neto según los especialistas es de $\$$ 63883374 el mismo que comparado con el Valor Actual Neto calculado según pronóstico CB de $\$ 72715279$ existe una diferencia de $\$ 9831905$ que bien puede ser considerado por los accionistas para la toma de decisión.

4. Se programaron 12500 pruebas para la simulación y $95 \%$ de confianza; este nivel se alcanzó cuando se ejecutaron 12457 pruebas y se detuvo el proceso.

5. Se tiene un $69,837 \%$ de certeza que el intervalo del Valor Presente Neto después de seis años se encuentra entre 20 y 80 millones de dólares. 
ISSN: 1794-9920 Impreso / Electrónico 2500-9338

Enero - Julio de 2019 - Volumen 19 Número 2, Año 2019 Págs. 70 - 80

6. Según el análisis de sensibilidad del Valor Actual Neto con Tasa de Descuento del 10\%, se encuentra que en los años 2019 y 2020 se consolida el negocio ITV con el $56.4 \%$.

\section{REFERENCIAS:}

Anderson, D. S. (2008). Estadística para la administración y la economia (10 ed.). México: Cengage Learning.

Monks, J. (1991). Administración de Operaciones. Mexico: Shaum.

Winston, W. (2005). Investigación de Operaciones (41418 ed.). Mexico: Cengage Learning. 